\title{
Unmasked diabetes insipidus after pericardial drainage and biopsy for pericardial effusion in association with Erdheim-Chester disease
}

\author{
John G. T. Augoustides, MD, FASE, ${ }^{a}$ and Wilson Y. Szeto, MD, ${ }^{\mathrm{b}}$ Philadelphia, Pa
}

$\mathrm{E}$ rdheim-Chester disease (ECD) is a rare form of histiocytosis that is characterized by the absence of Langerhans cells. ${ }^{1}$ Although long bone involvement is almost universal, more than $50 \%$ of patients also have extraskeletal involvement, including the orbit (exophthalmos), pituitary gland (diabetes insipidus), and pericardium (effusion). ${ }^{1,2} \mathrm{We}$ present a case of symptomatic pericardial effusion in a young woman with established ECD. After a subxiphoid pericardial window and biopsy under general anesthesia, the patient presented with severe diabetes insipidus in the postanesthesia care unit. To the best of our knowledge, this is the first report of this presentation as a perioperative manifestation of extraskeletal ECD.

\section{Clinical Summary}

A 27-year-old woman with established ECD presented with a symptomatic moderate pericardial effusion with echocardiographic features of cardiac tamponade. Because of her widespread ECD, she had bilateral exophthalmos and chronic bilateral femorotibial pain. She had never been given a diagnosis of diabetes insipidus.

The patient was scheduled for pericardial drainage, biopsy, and window creation to drain the symptomatic effusion, to obtain tissue for histology, and to prevent future effusions. After being kept nil per os for 6 hours, she underwent uneventful subxiphoid pericardial drainage and biopsy after achievement of general endotracheal anesthesia. A pericardial window was created to communicate with the right pleural space. Brisk urine output was noted intraoperatively in the absence of diuretic administration. After a smooth emergence from anesthesia, the patient was transferred to the postanesthesia care unit.

Her anesthetic recovery was complicated by massive diuresis at a rate of 1 to $2 \mathrm{~L} / \mathrm{h}$. The serum sodium level increased rapidly within 3 hours to a peak of $171 \mathrm{mmol} / \mathrm{L}$ (normal range, 135-145 mmmol/L), despite aggressive volume resuscitation.

In light of her known ECD and clinical presentation, a presumptive diagnosis of diabetes insipidus was made. Desmopressin therapy was added to the fluid replacement that was titrated to serial measurement of the serum sodium level.

From the Cardiothoracic Section, Anesthesiology and Critical Care, ${ }^{\mathrm{a}}$ and the Department of Cardiothoracic Surgery, ${ }^{\mathrm{b}}$ University of Pennsylvania School of Medicine, Philadelphia, Pa.

Received for publication Oct 10, 2007; revisions received Feb 21, 2008; accepted for publication March 2, 2008.

Address for reprints: John G. T. Augoustides, MD, FASE, Cardiothoracic Section, Anesthesiology and Critical Care, Dulles 680, HUP, 3400 Spruce St, Philadelphia, PA 19104-4283 (E-mail: yiandoc@ hotmail.com).

J Thorac Cardiovasc Surg 2008;136:217-8

0022-5223/ $\$ 34.00$

Copyright (C) 2008 by The American Association for Thoracic Surgery doi:10.1016/j.jtcvs.2008.03.005
The patient was transferred to the intensive care unit for ongoing management in consultation with nephrology and endocrinology. The serum sodium level was gradually normalized to avoid the clinical syndromes caused by cerebral edema.

After detailed questioning, the patient admitted to chronic polydipsia and polyuria on the order of 10 to $15 \mathrm{~L} / \mathrm{d}$. She had regarded this as normal because the onset of the diabetes insipidus had been gradual over several years. To date, she had been able to compensate accordingly. However, the syndrome was unmasked when she was made nil per os for her procedure after achievement of general anesthesia.

The remaining perioperative course was uneventful. The pericardial histology was consistent with ECD. After her surgical recovery, she underwent further inpatient chemotherapy for her primary disease. She was subsequently discharged home with maintenance desmopressin for her diabetes insipidus.

\section{Discussion}

This patient had compensated chronic central diabetes insipidus caused by pituitary involvement from her longstanding ECD. The perioperative diagnosis was made possible by the fluid restrictions required before elective general anesthesia. This is, to the best of our knowledge, the first report of its diagnosis in the perioperative period.

Central diabetes insipidus in ECD has a reported prevalence of $28.8 \%$. $^{1}$ The therapy of this syndrome in ECD has no distinguishing features. Central neurologic syndromes in ECD depend on the site of involvement. The most common areas are the pituitary, cerebellum, and pyramidal tracts. ${ }^{3}$ There are 3 described neuroradiologic patterns: infiltrative (44\%), meningeal (37\%), and composite with both infiltrative and meningeal features $(19 \%){ }^{3}$ Magnetic resonance imaging of our patient revealed an infiltrative pattern, with diabetes insipidus as the sole clinical neurologic manifestation.

Cardiovascular involvement by ECD can involve any of the major structures ${ }^{4,5}$ : pericardium (effusion), myocardium (systolic dysfunction, diastolic dysfunction, or both), endocardium (aortic regurgitation, mitral regurgitation, or both), conducting system (sinus node dysfunction), and major arteries (periaortic fibrosis and extrinsic renal artery fibrosis with secondary stenosis). Symptomatic cardiovascular involvement in ECD has been associated with a $31.4 \%$ mortality rate. ${ }^{4}$ In our patient the only detectable cardiovascular site of involvement was the pericardium.

The procedure of choice for surgical management of proved effusive pericardial effusion is transthoracic complete pericardiectomy. ${ }^{6}$ In our patient, however, we chose a subxiphoid pericardial window because this approach allowed drainage of the effusion, allowed us to obtain tissue for histology, and would make recurrence less likely. The created pleuropericardial window would not only prevent pericardial effusion with tamponade but also would allow thoracentesis as a future management option for recurrent effusion. The pericardial involvement in our patient might further regress because of her ongoing chemotherapy for ECD. 
Although the diagnosis of ECD was established in our patient already, the cause of the pericardial effusion was not proved. If this patient were to present with recurrent pericardial effusion despite ongoing appropriate chemotherapy for ECD, a more radical pericardial resection might be indicated because the pericardial histology was consistent with ECD. ${ }^{6}$

In summary, the clinical observation from this case is that ECD, although rare, has important cardiovascular presentations. Cardiac involvement might require surgical intervention. The associated systemic manifestations can complicate perioperative recovery. Successful management depends on taking the diverse clinical manifestations of this multicentric disease into account.

\section{References}

1. Veyssler-Belot C, Cacoub P, Caparros-Lefebvre D, Wechsler J, Brun B, Remy M, et al. Erdheim-Chester disease. Clinical and radiologic characteristics of 59 cases. Medicine (Baltimore). 1996;75:157-69.
2. Gupta A, Kelly B, McGuigan JE. Erdheim-Chester disease with prominent pericardial involvement: clinical, radiologic and histologic findings. Am J Med Sci. 2002;324:96-100.

3. Lachenal F, Cotton F, Desmurs-Clavel H, Haroche J, Taillia H, Magy N, et al. Neurological manifestations and neuroradiological presentation of Erdheim-Chester disease: report of 6 cases and systematic review of the literature. J Neurol. 2006;253:1267-77.

4. Haroche J, Amoura Z, Dion E, Wechsler B, Costedoat-Chalumeau N, Cacoub P, et al. Cardiovascular involvement, an overlooked feature of Erdheim-Chester disease: report of 6 new cases and a literature review. Medicine (Baltimore). 2004;83:371-92.

5. Elgeti T, Schlegl M, Nitardy A, Kivelitz DE, Stockburger M. Images in cardiovascular medicine. Magnetic resonance imaging guiding pacemaker implantation for severe sinus node dysfunction due to cardiac involvement in Erdheim-Chester disease. Circulation. 2007;115: e412-4.

6. Piehler JM, Pluth JR, Schaff HV, Danielson GK, Orszulak TA, Puga FJ. Surgical management of effusive pericardial disease. Influence of extent of pericardial resection on clinical course. J Thorac Cardiovasc Surg. 1985;90:506-16.

\title{
Intraoperative fluorescence angiography to determine the extent of injury after penetrating cardiac trauma
}

\author{
Nimesh D. Desai, MD, ${ }^{a}$ Fuad Moussa, MD, ${ }^{a}$ Steve K. Singh, MD, ${ }^{a}$ Peter Chu, MD, and Stephen E. Fremes, MD, ${ }^{a}$ \\ Toronto, Ontario, Canada
}

$\mathrm{P}$ enetrating cardiac trauma necessitates prompt surgical repair of injuries. We present a case report demonstrating the use of fluorescence coronary angiography to delineate the extent of coronary injury after temporary repair of a penetrating stab wound.

\section{Clinical Summary}

A 22-year old man sustained a knife wound to the left side of his chest and was transported to a quaternary care trauma center with severe shock and hypotension. Precipitous loss of vital signs while in the hospital led to immediate left anterior thoracotomy in the

From the Divisions of Cardiac and Vascular Surgery, ${ }^{\mathrm{a}}$ and General Surgery, ${ }^{\text {b }}$ Sunnybrook Health Sciences Centre, University of Toronto, Toronto, Ontario, Canada.

Received for publication May 12, 2007; accepted for publication May 30, 2007.

Address for reprints: Nimesh D. Desai, MD, Division of Cardiac and Vascular Surgery, Sunnybrook Health Sciences Centre, 2075 Bayview Ave, Room H410, Toronto, Ontario, Canada, M4P 1S7 (E-mail: nimesh.desai@vtoronto. ca).

J Thorac Cardiovasc Surg 2008;136:218-9

$0022-5223 / \$ 34.00$

Copyright $\odot 2008$ by The American Association for Thoracic Surgery doi:10.1016/j.jtcvs.2007.05.073 emergency department, revealing a tense hemopericardium. The pericardium was incised sharply, and a large amount of clot was evacuated. Spontaneous rhythm returned after a brief period of open cardiac massage. Examination of the heart revealed a through-and-through laceration of the right ventricle, with an anterior wall entry wound and posterior wall exit wound. Surgical staples were applied to entry and exit wounds for immediate hemostasis, and the patient was transferred to the operating room for definitive cardiac repair.

Rapid biventricular failure with hemodynamic instability ensued, although a transesophageal echocardiogram revealed no intracardiac injuries. In response to impending circulatory arrest, cardiopulmonary bypass was rapidly initiated. Examination of the posterior wall exit wound during bypass revealed that the surgical staples had occluded the posterior descending coronary artery (PDA). The staples impinging on the PDA were removed. The posterior ventricular laceration was in close proximity to the PDA but did not involve the artery itself. Ventricular repair was completed with pledgeted polypropylene sutures, as illustrated in Figure 1. It was unclear whether the PDA had been significantly damaged by the staples, and coronary artery bypass grafting to the PDA was considered. To delineate the coronary anatomy, intraoperative coronary fluorescence angiography was performed. ${ }^{1,2}$ A bolus of $1 \mathrm{~mL}$ indocyanine green contrast dye was injected into the aortic root through the cardioplegia catheter. As shown in Figure 2, the PDA was widely patent, and excellent perfusion of the posterior wall was 